\title{
Innovation and Analysis of Police Riot Helmets with Continuous Textile Reinforcement for Improved Protection
}

\author{
C. Roedel and X. Chen ${ }^{+}$ \\ School of Materials, University of Manchester, Manchester, UK
}

( Received 4 October 2006, accepted 4 February 2007)

\begin{abstract}
At the present there exist two different types of riot helmet shells, a thermoplastic and a composite shell. The composite shell is believed to be superior in impact performance, but more expensive and complicated in manufacturing partially due to the fact that the textile reinforcement material has to be cut into pattern pieces before they can be applied to the helmet shaped mould. However, the discontinuation of fibres is likely to reduce the level of protection and shorten the lifetime of a helmet. The aim of this research is to develop a helmet shell consisting of a single-piece of fabric without creating wrinkles and without the necessity of cutting the fabric. For this purpose, a new type of woven fabric has been developed and an apparatus has been set up to drape the fabric to a helmet shell. The helmet shell was manufactured using a single piece of Kevlar woven fabric. In parallel the finite element method is applied to investigate the shock absorption behaviour of the helmet shell made from both multi-piece and single-piece fabric reinforcements. The results show clearly the advantage of the single-piece helmet shell over its existing multi-piece counterpart.
\end{abstract}

Keywords: police helmet, composites, finite element analysis

\section{Introduction}

Riots are common in many countries with increasing trend. The riot police officers are exposed to a large number of hazardous threats from the rioters. Rioters pick up anything they can such as metal pieces, stones, bats, knifes, and even petrol bombs to attack police officers. In addition, it is often the case that direct body contacts take place between the police officer and rioters during foot pursuit or restraint. In view of these hazards it is clear that helmets that provide improved protection will be necessary. Generally speaking, a helmet with improved performance should be more protective, lightweight, comfortable to wear and cost effective. However, improved impact protection often comes with side effects such as higher weight and discomfort. This research project concentrates on the innovation and analysis of a new type of composite riot helmet shell.

Approximately a total of 10,600 police riot helmets are sold to the UK police annually and 40,000 helmets were in use throughout the UK in 2003, which verifies that there is a continuous need of riot helmets. At the present there are two types of riot helmets available, a thermoplastic and a textile-reinforced (duroplastic) helmet shell. The technology remains the same since decades. While the riot helmets made of thermoplastics currently dominate the market in the UK due to its low costs, police helmets made of a composite shell are gaining ground due to their better impact performance and have been recognized and promoted by the Home Office Scientific Development Branch (HOSDB), who certifies police protection equipment. The aim of this research is to develop a continuous reinforced composite shell using a single piece of fabric as reinforcement and to evaluate the performance of such made helmet. Commonly used composite helmets are made of trimmed fabrics, which are consolidated using the hand-lay up method. Before applying the fabrics to the mould, these have to be pre-cut into various geometries, which are then impregnated with an activated resin and applied to pre-defined areas of a negative type mould.

Main materials used are fabrics consisting of aramid and glass fibres. The disadvantage of the present hand-lay-up procedure for composite shells is the number of processing steps, waste of material, time consumption and hence high costs. However, one of the main disadvantages of the existing composite shells is their discontinuous reinforcement, which may reduce the impact performance and results into failure

\footnotetext{
${ }^{+}$Corresponding author. Tel.: +44-161-306 4113, E-mail address: xiaogang.chen@manchester.ac.uk
} 
modes such as matrix cracks and delamination. This paper reports on the characterisation of helmet shells made from multi-piece and single-piece fabric reinforcements using finite element methods (FEM), based on flat panels and helmet shells.

\section{Literature Review}

It must be said that not much has been reported on practical and theoretical investigation about the police riot helmets. Generally, most information about non-ballistic helmets is found about motorcycle and sports helmets, which deal predominantly with the improvement of the energy absorption by optimising the density of the absorbing protective padding layer underneath the shell. The riot helmet analysis proposed in this paper aims to improve the impact performance of the riot helmet shell, which can be determined considering performance indices such as the amount of absorbed impact energy, contact forces, acceleration, and the material damage inside the helmet. A comparison of the riot police helmets with motorcycle or sports helmets is difficult to be carried out mainly due to the fact that the helmet design is different. In terms of undercut designed motorcycle helmets, which are similar to riot helmets, there exist some information about practical work using helmet drop tests and theoretical work based on simple finite element simulation.

Gilchrist and Mills [1] studied the structural behaviour and deformation mechanism of thermoplastic and composite motorcycle helmet shells. They showed that the composite shell absorbed significantly more energy than the thermoplastic shell and that the rebound velocity of composite shells is smaller due to delamination. This verifies that composite shells are more advantageous in comparison to thermoplastic shells. In another motorcycle related study Yettram et al [2] proposed a FE helmet model simulation to investigate the impact performance behaviour of the shell and absorbing liner. To understand the impact performance they modified the shell stiffness and the protective padding density. The conclusions of their study were that the lower the density of the protective padding the more impact energy can be absorbed, and that the helmet shell is not allowed to be too stiff to enable energy dissipation to the absorbing liner. However, such work about composite helmets does not consider the composite design of the helmet shell, which is an important component.

Dionne et al reported on the overall impact performance of commercially available riot helmets [3]. Six helmets of different manufacturers have been impact tested laterally, at the front and at the visor. Results showed that the helmet impact performance strongly depends on the type of impact, whether it is rounded or edged. Detailed information about exposed energy values of various impactors had been provided. It was proposed that the protection of riot helmets ends at the average impact energy of a half brick, which lays approximately at 175J. Furthermore, it was proposed that the lateral helmet areas withstand higher impact energies than the frontal area, and that the face shield absorbs much less impact energy than the helmet shell. However, this work did not investigate the failure mode of the laminated composite shell. Police riot helmets have predominantly to be designed to protect against impact energies up to approximately $120 \mathrm{~J}$ and to produce not more than a resultant acceleration of 250'g', this has been suggested in several riot helmet test standards and is considered in this paper $[4,5,6]$. Such impacts fall into the category of the low-velocity impacts, which can cause damages of the composite compound, which may not be visible with the naked eye. These failure modes are known as matrix cracking caused by bending or shear, delamination and fibre breakage. In the case of matrix cracking and delamination, the impact performance of the composite helmet shell can be severely reduced, making the helmet less protective.

Research results have been reported on the study of the delamination phenomenon of laminated composites, which is directly related to the riot helmet investigation. Choi et al carried out experiments on graphite/epoxy prepregs to investigate the damage behaviour after a low-velocity impact. For that purpose they developed an impact tester, which consists of a line-nosed impactor. Due to the fact that the impact damage within the composite structure is not visible with the eye, they used X-Ray microscope to investigate the structure. From their experiments they found that there are three failure modes after an impact, namely critical matrix cracks, delamination and micro cracks. Their experiments showed that delamination is always accompanied by a critical matrix crack. The performance was found to be dependent on the stacking sequence of the laminates [7]. They continued their work into theoretical analysis [8] by focusing on mechanical modelling of the composites. The aim of the analysis was to provide knowledge about the initial failure mode with its location and the initiation of delamination. They developed a matrix failure criterion, which is the first failure mode beside delamination and micro cracks according to them. The results of the predictions agreed very well with experimental results. They concluded that matrix cracking is the first failure mode in the composite laminates, which causes delamination and with occurring delamination micro- 
cracks. The matrix crack depends on the interaction of the interlaminar shear stresses/strength and the inplane tensile stresses/strength. Furthermore the growth of delamination is dominated by the out-of-plane normal stress and the interlaminar shear stress. Aslan et al [9] studied the impact performance of five layer Glass/Epoxy plates exposed to a hemispherical impactor under low velocity impact. They used an experimental impact apparatus and a numerical transient dynamic finite element analysis code 3DIMPACT. Performance indices were the stresses and contact forces obtained during impact. For the evaluation of impact failure they used two criterions, namely, the critical matrix cracking criterion and the impact-induced delamination criterion proposed by Choi et al $[7,8]$. With the help of the numerical simulation and these criterions they were able to predict delamination failure and size in the composite plates.

$\mathrm{Li}$ et al carried out studies on continuous fibre-reinforced composite laminates made of cross-ply $0 \% / 90^{\circ} / 0^{\circ}$ composite plates [10]. They believe that shear matrix cracks close to the impacted area are caused in the second $90^{\circ}$ layer, these cracks initiate delamination along the bottom or upper interface of the cracked layer. A long bending matrix crack can be caused in the third $0^{\circ}$ layer resulting in further delamination. These failure modes were considered by using numerical techniques. For that purpose they proposed an FEM code [11], where they verified the developed numerical model by comparison with experimental results including results from other researchers. Their comparisons are based on carbon/epoxy composite laminates of the stacking sequence $0^{\circ} / 90^{\circ} / 0^{\circ}$. They investigated various aspects such as the length of the composite laminate, the boundary conditions of the laminate, impact velocity, impactor mass, and the relationship to the individual damage modes, namely, delamination size, matrix crack distribution, force-time history and the central displacement. In terms of delamination size there exists an increase in delamination with a decrease in thickness of the $90^{\circ}$ layer. Where the delamination extension increases faster into the laminate length than into the width. Generally, matrix cracks are similar to the delamination shapes, whereas bending cracks are few and extended and shear cracks are numerous and short. When the thickness of the $90^{\circ}$ laminate layer is decreased the number of shear cracks and the length of bending cracks increases, because the stiffness in the $90^{\circ}$ layer is reduced. It is worth mentioning that matrix cracks occur before delamination. In terms of forcetime and deflection behaviour of the composite laminate, the stacking sequence within the composite plate had no significant influence. Furthermore, they found that an increase in impact velocity at identical impact energies (mass and velocity is varied), the impact force increases and impact duration becomes shorter. At the same time with increasing impact velocity the matrix cracks and delamination appear earlier. Whereas the central deflection of the plate increases with increasing mass. The increase of impact velocity seemed to have a larger effect on the delamination size than the increase in impactor mass at same impact energies. The results obtained from their FEM model were in good agreement with the results obtained from practical experiments.

\section{Finite Element Modelling}

In order to analyse the impact performance of textile reinforced riot helmet shells reinforced by singlepiece and multi-piece fabrics, models for FE modelling are set up. Multi-piece and single-piece FE plate models were designed as a first step to investigate the impact performance. The obtained data can be related to the riot helmet analysis in a later study. Furthermore, FEM was used to design single-piece helmet shells for impact analysis with different objects. Furthermore, a method to produce continuous textile reinforced riot helmet shells has been established and is briefly introduced in this paper.

\subsection{FEM analysis of composite panels with single-piece and multi-piece reinforcements}

To evaluate the impact performance of textile composites reinforced by single-piece and multi-piece fabrics, FE models of flat composite panels of various designs were set up. The purpose of this analysis is to investigate how fabric overlapping in composites would influence the impact performance of the composite. Five different plate models, four with multi-piece reinforcements and one with single-piece reinforcement, were created. The four different multi-piece models were designed to have overlapping length of $20 \mathrm{~mm}$, $30 \mathrm{~mm}, 40 \mathrm{~mm}$ and 50mm respectively and are accordingly denoted as MP20, MP30, MP40, and MP50. The design of multi-piece plates is illustrated in Fig. 1. The one single-piece plate model was created to have the same overall width as the multi-piece plates and it is referred to as SP. 


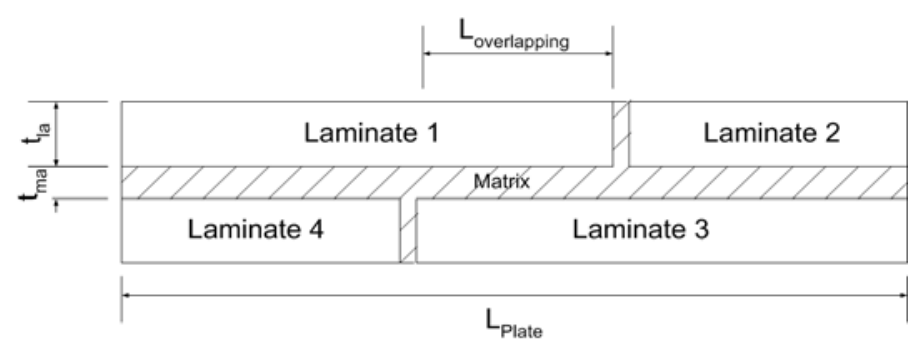

Fig. 1: Illustration of FE multi-piece plate design

The size of all the plate models has the same dimension with the width being $50 \mathrm{~mm}$, length $200 \mathrm{~mm}$, and thickness $2.4 \mathrm{~mm}$. The different models were impacted by a circular bar of $0.486 \mathrm{~kg}$ at the centre of the plates with four different impact energies (20J, 50J, 80J, and 110J). This is due to simulate the low speed impact, which is most likely to be encountered in riot situations. FE software MSC.Marc Mentat was used to carry out the analysis. The performance of the composite panels in displacement, residual deformation, total strain energy and different plastic strain components has been considered.

Fig. 2 shows the displacement and residual deformation of the single-piece plate and a representing multi-piece plate (MP50) when impacted with four different impact energies. It is clear that high impact energy causes larger displacement and residual deformation for both the multi-piece and single-piece plates and that the displacement and residual deformation for MP50 are larger than that of SP. The latter indicates that a helmet shell made from a single-piece will be indented less than a helmet shell made from a multipiece fabric, and would therefore be more able to block the impact force be transmitted to the head.

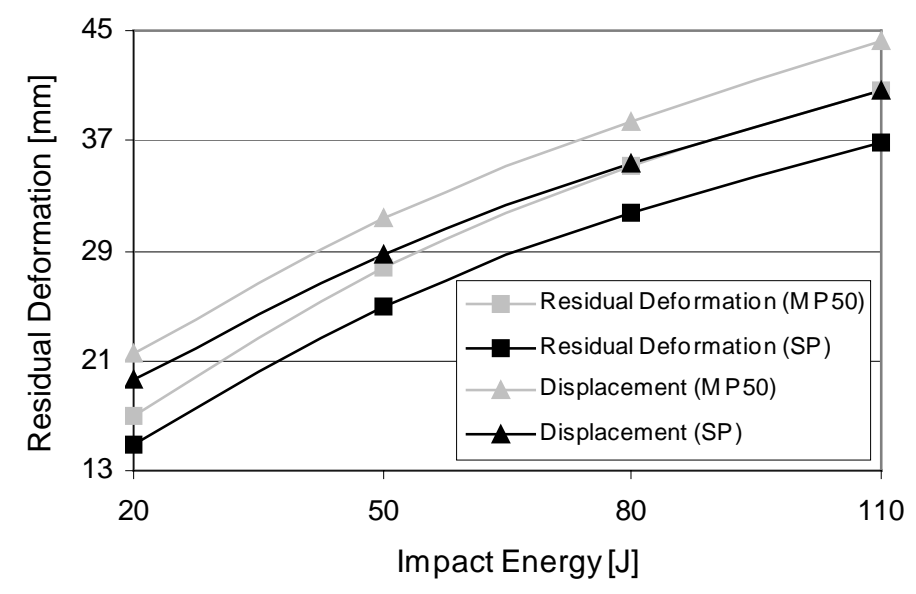

Fig. 2: Residual deformation of plates at different impact energies

Fig. 3 illustrates the influence of the overlapping length on the total strain energy when the panels are impacted with different levels of impact energy. It is evident that higher impact energy leads to higher total strain energy. It is also noticed that at the same impact energy level the total strain energy decreases slightly as the overlapping length increases, with the single-piece plate having the lowest total strain energy. This suggests that a composite panel with shorter overlapping length is able to absorb more impact energy because it is easy to be deformed, whereas when the overlapping length is long and in the case of a singlepiece fabric reinforcement, the composite panel is less able to absorb impact energy. Combined with the discussion about Fig. 2, it can be said that helmets reinforced by a single-piece reinforcement are more likely to block the impact force at the impacting location, but because of being less able to absorb impact energy, though only slightly, the impact force may work to swing the head creating a higher acceleration of the head. In helmet engineering, blocking impact from transmitting to the head and reducing the acceleration of the head are two important targets. Optimisation of a helmet shell material is needed to protect the head. However, it seems that at lower impact energy where the main problem is transmitted force, the helmet shell made from a single-piece reinforcement may provide a better solution. 


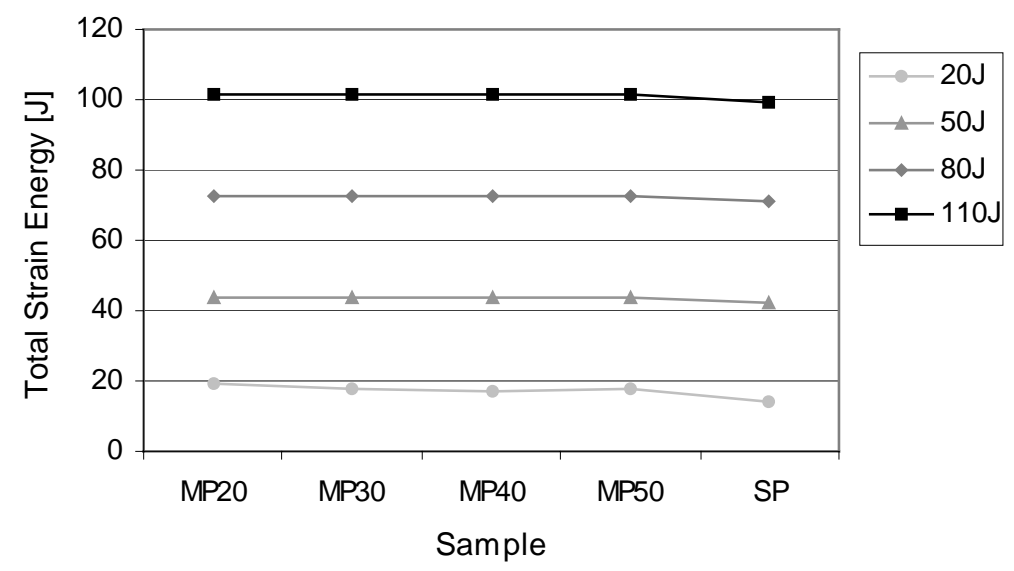

Fig. 3: Total strain energy absorbed after different impact energies

Another reason for the single-piece plate to have lower displacement and residual deformation than the multi-piece plates could be that multi-piece composite structures are able to absorb energy by various failure modes such as matrix cracking and therefore delamination. It is evident from Fig. 4 that there is higher strain in places where there is no reinforcing fibres. The simulations showed that the strains inside the MP plates are higher when compared with the SP plate.

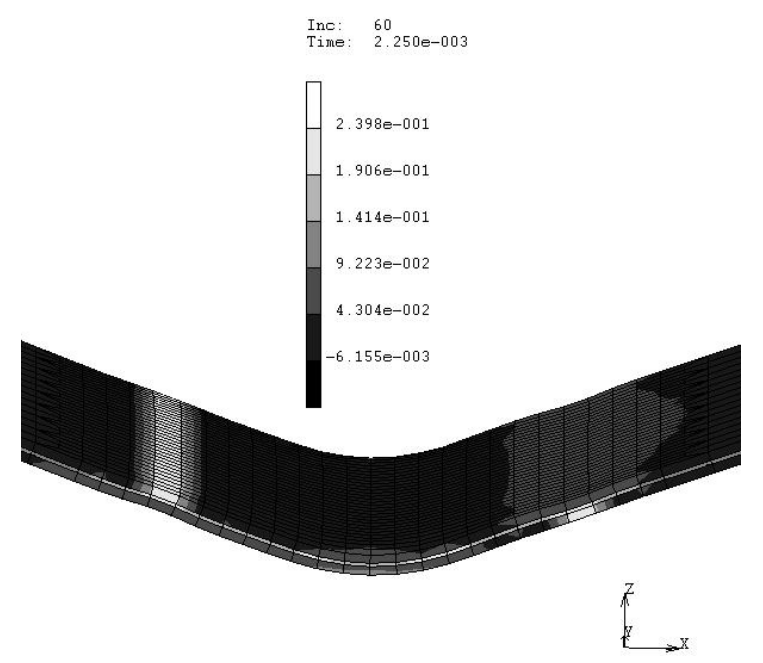

Fig. 4: Typical visual image of multi-piece (MP40) plate and plastic strain distribution

In order to provide overall information about strain distribution inside the plate, reference points along the centre axis in equal distances were chosen for comparison, and this is displayed in Fig. 5. The term " $0 \mathrm{~mm}$ " stands for the location of the impact point.

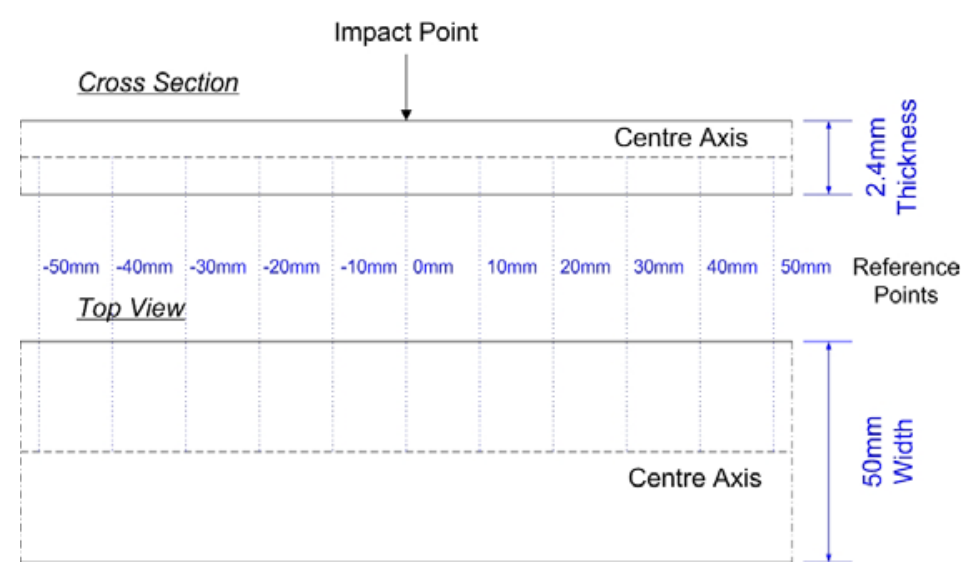

Fig. 5: Reference points chosen along the centre axis of the plates 
Representative trend curves of an 80J impact for different plastic strain components, $\mathrm{e}_{11}, \mathrm{e}_{33}$, and $\mathrm{e}_{31}$ are illustrated in Fig. 6. Again, due to similarity the result of multi-piece model MP30 is illustrated. In Fig. 6., $\mathrm{e}_{11}$ is defined as the plastic strain along the plate length, $e_{33}$ is the plastic strain normal to the plate, and $e_{31}$ is the shear plastic strain. It is observed that after the impact shear stress causes the highest plastic strain beside the impact centre. It is also evident that there is much lower plastic strains in SP than in MP30. The same trend has been observed for all other multi-piece models and all other impact energies. It can be seen that the strain fluctuation of the SP is smoother and lower than that of the MPs. Furthermore, the simulations show that the highest strains for multi-piece plates take place approximately $10 \mathrm{~mm}$ to $30 \mathrm{~mm}$ next to the impact point. The significant difference in responding to the impact by the single-piece and multi-piece models calls for further investigation.

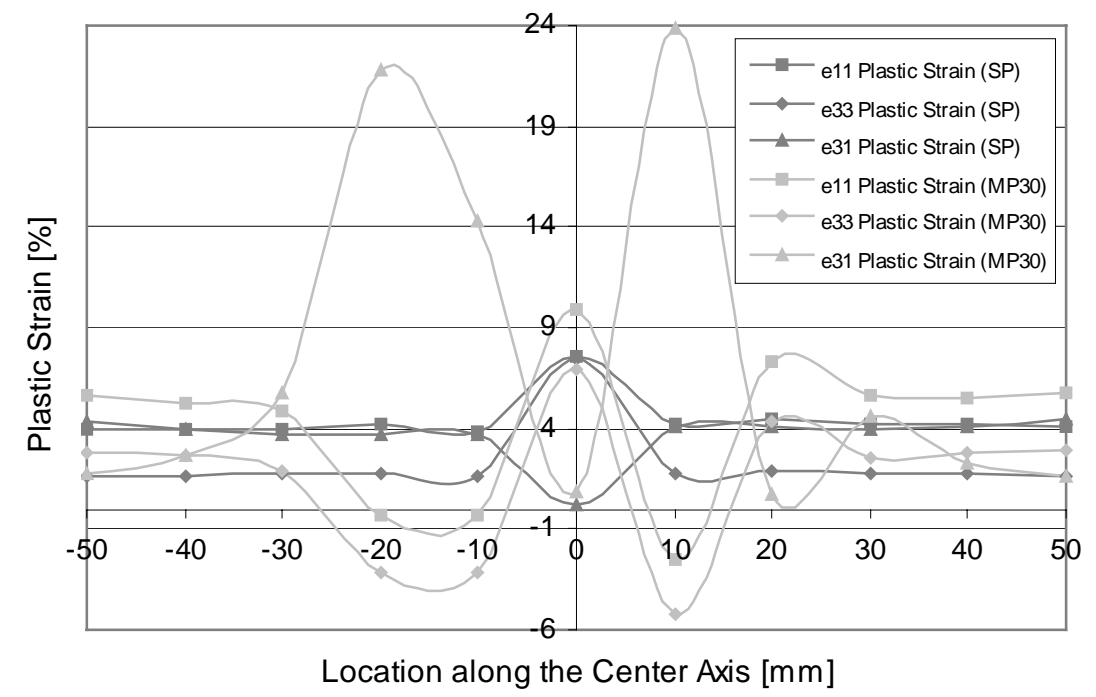

Fig. 6: Comparison of plastic strain distribution along the centre axis of SP and MP30 after 80J impact

Fig. 7 shows the shear plastic strain caused by impact with different impact energy for the multi-piece and single-piece plates. As can be seen, the increase of impact energy results in increase of plastic shear strain, and the increase in overlapping length leads to a reduced shear plastic strain at all levels of impact energy. This result implies that when the overlapping length in a composite plate is small, the material is most likely to delaminate. Increase the overlapping length is effective in preventing delamination. In the case of the single-piece plate, the material will remain integrated as long as the fibres are not damaged. This suggests that a helmet shell made from single-piece fabric is more likely to remain intact than the multi-piece counterparts.

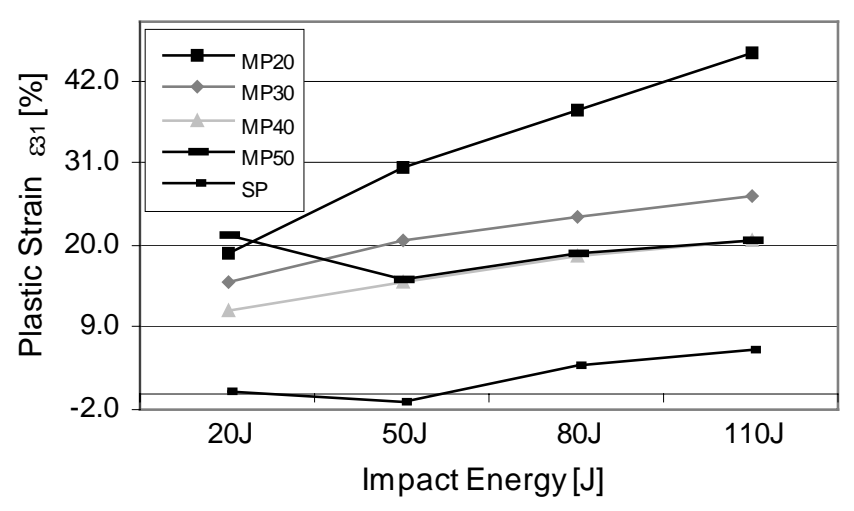

Fig. 7: Shear strain trend for different plate designs

From the modelling of flat composite panels, it can be concluded that composites reinforced by a singlepiece fabric provide improved impact performance in terms of reduced displacement, deformation and shear strain distribution inside the composite. Riot helmet shells reinforced by a single-piece fabric will perform 
better in blocking impact force, whereas helmet shells reinforced by multi-piece fabrics can help to reduce the head acceleration caused by the impact.

\subsection{Geometrical modelling of a riot helmet shell}

The geometrical helmet model was created using MSC. Marc based on digitised coordinate values. The data is taken from the British headform standard called "Headform for use in the testing of protective helmets" [12], which was converted into three-dimensional (3D) coordinates. The headform coordinates are expanded to the dimensions of a geometrical riot helmet shell, with a grading factor calculated from previously measurements from a commercial riot helmet. Two-dimensional (2D) quadrilateral elements were used in the creation of the geometrical models of the helmet. Dispensable finite elements were deleted. The edges of the shell were formed using the measurements taken from a commercially available riot helmet. The thickness of the helmet shell was created by these of 3D hexahedral elements. Fig. 8 shows a such created geometrical model of a riot helmet shell.

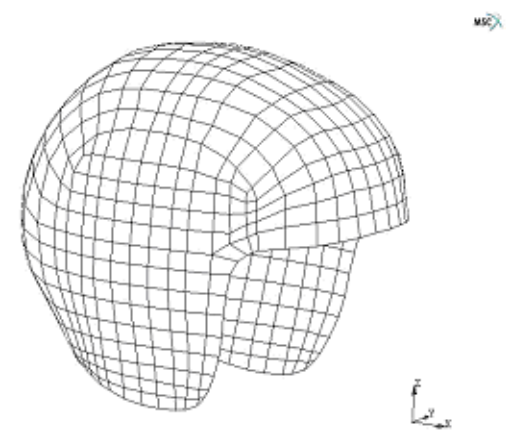

Fig. 8: Geometrical model of a riot helmet shell

\subsection{Impact Performance of Riot Helmet Shell}

A 3D finite element impact model of a riot helmet shell was set up and impacted on by impactors of different geometrical shapes. The Single-Step Houbolt operator was applied as the default time integration method for the dynamic transient impact analysis. Element types 134 for tetra and type 7 for hex elements were used in the meshing process. The helmet shell design was considered to be with single-piece reinforcement and defined with Kevlar/Epoxy material properties, which were derived from earlier tensile tests. In order to simplify comparison each impactor was defined with steel material properties and the volume was kept constant to enable equal mass of $0.488 \mathrm{~kg}$. Three different objects were considered, which are a ball, a bar and a brick, representing the commonly encountered riot threats. In this part of the research, four different impact energy levels were used (20J, 50J, 80J, 110J) to represent low-velocity impact. The impact in this simulation occurred at the helmet side area and the shell was constrained at its edges to enable secure fixation and sufficient flexibility to respond to the impact. Performance Local displacement and residual deformation, contact force, helmet acceleration, and total strain energy are used as the performance indices of the helmet shell. The data was collected from the initial contact points of both the shell and the impactor. The use of the riot helmet shell is to prevent excessive impact force from being transmitted to the head and to absorb the shock created by the impact to minimise the acceleration caused to the head. Fig. 9 shows the total displacement and the amount of residual deformation of the shell after the impact. The displacement is defined by the deflection of the initial contact point of the helmet shell and the deformation is the residual deflection after the impact. It can be seen that both the displacement and residual deformation increase nonlinearly with increasing impact energy inside the low-velocity impact range set up in this simulation. It is evident that for each level of impact energy the ball impactor causes higher displacement and residual deformation than the bar and brick, and it is believed to be due to the smaller contact area. The elasticity percentage of the helmet shell is presented at the top of the columns. It is clear that the elasticity of the helmet shell decreases with the increase in impact energy. The gap between the displacement and residual deformation values becomes smaller with increasing impact energy. This indicates that a higherenergy impact will tend to leave the helmet shell more permanent damage as can be expected. 


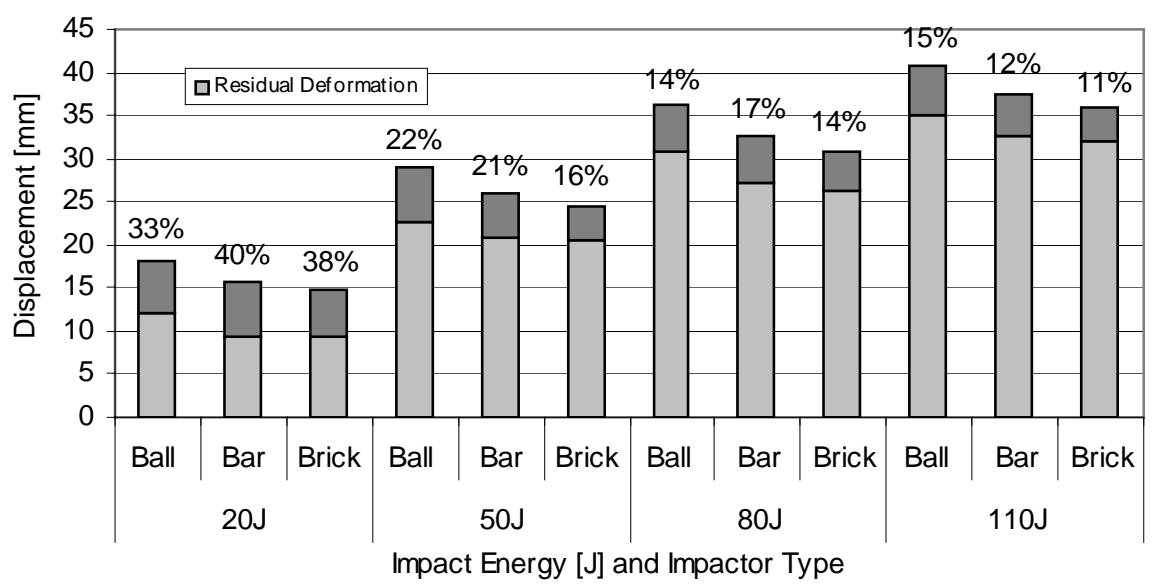

Fig. 9: Displacement and residual deformation caused after different shaped impactors

The displacement of the helmet shell is a mechanism for impact energy absorption, which is reflected by the value of total strain energy during the impact process. One of the key aims of the riot helmet is to absorb as much of the incoming impact energy as possible as long as the transmitted impact force to the head is under control. Fig. 10 displays the influence of impact energy levels on the total strain energy through the FE simulation. It is as expected that higher level of impact energy cause higher total strain energy. It is interesting to see, however, that the percentage of impact energy converted to total strain energy is smaller at lower levels of impact energy level than at higher ones. This shows that at lower levels of impact energy the single-piece helmet shell is more likely to bounce the impact off and when the impact energy is higher, the helmet shell is more likely to deform to absorb the impact energy. These two modes work together for the durability and protection of the helmet shell, which are both needed for the helmet engineering.

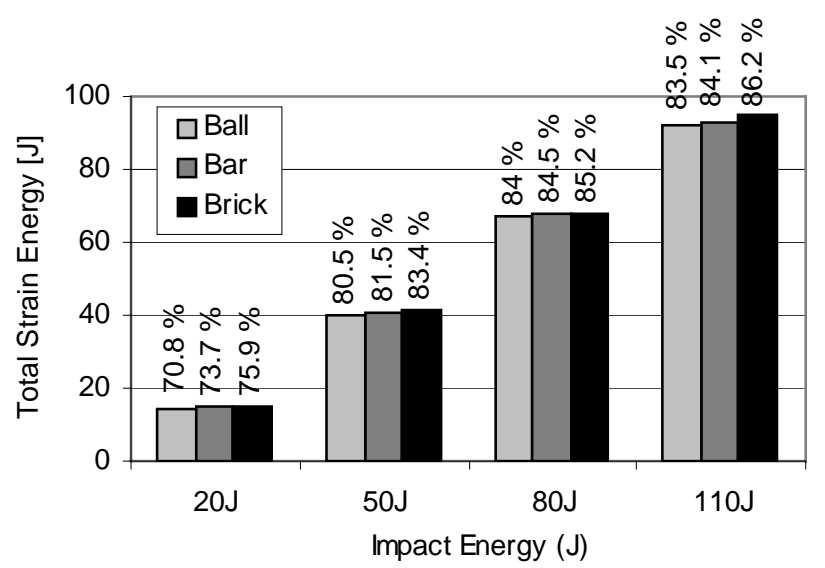

Fig. 10: Total strain energy at different impact energy levels and impactors

Fig. 11 shows a typical stress distribution over the helmet shell when impacted by a brick. It is seen that the highest normal stresses on the shell occur especially at the pointed corners of the impactor. These high stress areas are more prone to various failures. More damage can be brought to the helmet shell if the impact lands on the helmet shell with the pointed edge. For a helmet shell with discontinuity of the reinforcement material, a sharp edge impactor could lead to failure of the helmet shell such as matrix cracks and delamination. This will be the focus of further FE simulation of riot helmet shells reinforced by multi-piece textiles.

As mentioned already, the acceleration caused to the helmet during impact is an important factor in helmet engineering. High accelerations to the helmet create a rotation of the brain and can cause blunt trauma or in extreme cases even lead to the disruption of the neural tissue. The acceleration is mainly reduced by the protective padding underneath the helmet shell, which is made of a rigid and expandable material such as Polystyrene. The shell plays a secondary role but also has to support the reduction of 
acceleration. Generally, the acceleration values are decreasing from the outer shell in the direction to the head. However, the FEA analysis showed that the shapes of the impactors did not significantly affect the acceleration caused by the three impactors. The analysis of a continuously reinforced riot helmet shell has provided information about various performance indices. Future work will focus on the analysis of discontinuously reinforced helmet shells and their comparison with the single-piece shell.

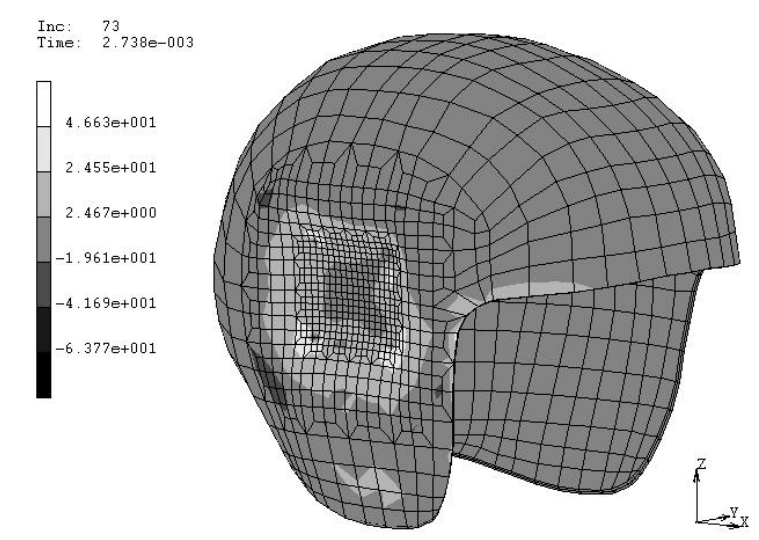

Fig. 11: Normal stress distribution over the helmet shell after side impact

\section{Manufacturing of continuous textile-reinforced riot helmet shell}

A moulding apparatus has been developed enabling the conformation of a single-piece of woven fabric to a positive mould of headform. Existing moulds for producing composite riot helmet shells are of the negative type to suit the patching process of the trimmed fabrics. However, it was found difficult to use a negative mould in this study, because the fabric has to be draped to the mould surface by applying forces to enable smooth conformation. At the same time the surface of the fabric can be checked on wrinkles or unsmooth areas. Therefore, it was decided to develop a positive mould apparatus. An important issue of the positive mould design is the removal of the cured undercut designed shell. Due to the dimensions of the helmet shell the removal of the cured shell is stopped by the decreasing circumference at the lower part of the shell. To facilitate the removal, the mould has to be supported by a collapsing mechanism, which prevents any bending to reduce damage risk of the matrix. The dimensions for the positive mould apparatus have been determined using a plaster print-up of a commercial riot helmet shell. The plaster print-up provided a smooth inner surface, which was covered with resin soaked glass fibre mats until an adequate thickness was achieved. After curing, the edges were cut and the mould was accurately divided into three parts, which consisted of two symmetric identical outer mould parts. The middle part was attached to a stand and provided fixing mechanisms for the two outer parts. Fig. 13 shows a completely conformed woven fabric to the moulding apparatus. Considering the marked lines, high shear of the fabric occurs at the front and back edges, but wrinkles were successfully avoided.

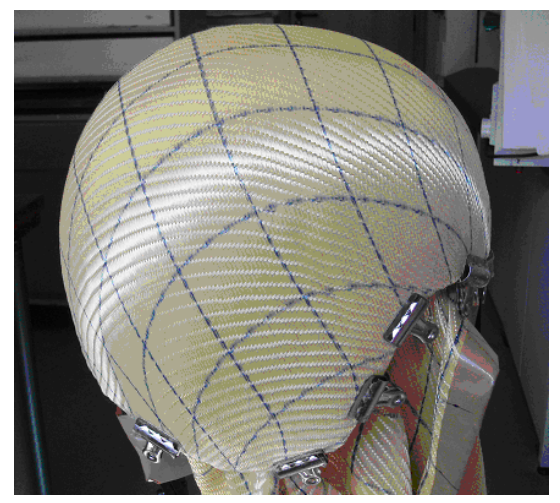

Fig. 13: Conformed woven fabric over helmet mould

\section{Conclusion and further work}

This paper presented the FE simulation of composite plates with continuous and discontinuous textile 
reinforcements to show the difference in impact performance. FEA has been carried out on continuous reinforced riot helmet shells including the methodology of designing a riot helmet shell. Furthermore, a new technology of producing continuous reinforced police helmet shells and their creation using a collapsing mould apparatus has been demonstrated.

The experiments carried out on composite plate models showed that single-piece plates performed better in terms of displacement and residual deformation. The strain distribution inside the composite structure, which are responsible for various failure modes such as matrix cracks and delamination, were higher for the multi-piece plates for three different vector orientations. This gives evidence that continuous reinforced composites are less prone to failure modes and provide improved impact performance. Further research will be continued on the analysis of discontinuous reinforced riot helmet shells. For that reason impact simulation has been carried out on continuous reinforced helmet shells including the methodology of designing a riot helmet shell. The impact analysis using different shaped impactors served as a first step to understand the impact response of the shell against different objects. Impact parameters such as displacement, residual deformation, total strain energy absorbed and stress distribution along the surface have been considered. Further work will concentrate on the impact performance of multi-piece and single-piece helmet shells considering stresses and strains in the composite structure to verify that single-piece helmet shells provide enhanced protection.

\section{References}

[1] A. Gilchrist. Impact deformation of ABS and GRP motorcycle helmet shells. Plastics, Rubber and Composites Processing and Applications. 1994, 21: 141-150.

[2] A. L. Yettram, N.P.M.G., B.P. Chinn. Materials for motorcycle crash helmets - a finite element parametric study. Plastics, Rubber and Composites Processing and Applications. 1994, 22: 215.

[3] J. P. Dionne, I. El Maach, A. Shalabi, and A. Makris. A Method for Assessing the Overall Impact Performance of Riot Helmets. Journal of Applied Biomechanics. 2003, 19: 246-254.

[4] HOSDB Protective Headwear Standard for UK Police. 2004.

[5] Z. Aslan, R. Karakuzu, B. Okutan. The response of laminated composite plates under low-velocity impact loading. Composite Structures. 2003, 59: 119-127.

[6] CSA Riot Helmet Standard. CSA Z611-M86. Toronto: Canadian Standard Association. 1986.

[7] NIJ Standard for Riot Helmets and Face Shields. NIJ Standard 0104.02. Washington, DC: U.S. National Institute of Justice. 1984, Oct..

[8] H.Y Choi, R. J. Downs, F. K. Chang. A new approach toward understanding damage mechanisms and mechanics of laminated composites due to low-velocity impact: part I-experiments. J Compos Mater, 1991, 25: 992-1011.

[9] H.Y Choi, R. J. Downs, F. K. Chang. A new approach toward understanding damage mechanisms and mechanics of laminated composites due to low-velocity impact: part II-analysis. J Compos Mater. 1991, 25: 1012-38.

[10] Z. Aslan, R. Karakuzu, B. Okutan. The response of laminated composite plates under low-velocity impact loading. Composite Structures. 2003, 59: 119-127.

[11] C.F. Li, N.H., Y.J. Yin, H. Sekine, H. Fukunaga. Low-velocity impact-induced damage of continuous fiberreinforced composite laminates. Part I. An FEM numerical model. Composites: Part A. 2002, 33: 1055-1062.

[12] C.F. Li, N.H., J.G. Cheng, H. Fukunaga, H. Sekine. Low-velocity impact-induced damage of continuous fiberreinforced composite laminates. Part II. Verification and numerical investigation. Composites: Part A. 2002, 33: 1063-1072.

[13] BS EN 960:1995. Headform for use in the testing of protective helmet. 1995. 